\title{
LA LETTERATURA ITALIANA TRADOTTA IN ArgenTina
}

\author{
Silvia Catton ${ }^{*}$
}

\begin{abstract}
In due secoli di storia della letteratura argentina, lo sviluppo della letteratura italiana tradotta è in stretto rapporto con i propositi pedagogici o estetici determinati dal contesto culturale dei diversi momenti storici. In linea di massima, è possibile affermare che, dalla conformazione dello Stato nazionale e durante $\mathrm{i}$ primi decenni del XX secolo, la traduzione letteraria mirava ad ampliare l'orizzonte culturale di un lettore che si consolidava al ritmo della fiammante nazione. Nelle fasi successive e in stretto rapporto con la politica culturale portata avanti da Victoria Ocampo a partire dal 1931 tramite la rivista Sur e il suo posteriore progetto editoriale, la traduzione è stata soprattutto una pratica di scrittura che ebbe un'influenza decisiva nell'ordito della letteratura nazionale favorendo il suo rinnovo e incentivando le versioni di traduttori argentini. Fu questa un' apertura che favorì, durante la seconda metà del secolo, nel contesto della ricezione della letteratura universale, l'ingresso della letteratura italiana in Argentina. Il presente lavoro tratta in maniera sistematica, ma provvisoria, il panorama della letteratura italiana tradotta in Argentina. Lo scopo principale comporta il registro dei momenti più fecondi e l'interpretazione degli esiti ottenuti nei confronti della traduzione nel sistema letterario nazionale.

PAROLE CHIAVE: letteratura italiana, letteratura argentina, traduzione, fasi di sviluppo
\end{abstract}

RESUMO: Em dois séculos de história da literatura argentina, o desenvolvimento

* Universidad Nacional de Córdoba, Córdoba (Argentina) - cattonisilvia@gmail.com Processo n.2013/20971-0 Fundação de Amparo à Pesquisa do Estado de São Paulo (FAPESP)

DOI: http://dx.doi.org/10.11606/issn.2238-8281.v0i34p90-102 
da literatura italiana traduzida está em estreita relação com os propósitos pedagógicos ou estéticos determinados pelo contexto cultural dos diversos momentos históricos. De modo geral, é possível afirmar que, desde a formação do Estado nacional e durante as primeiras décadas do século XX, a tradução literária visava ampliar o horizonte cultural de um leitor que se consolidava ao ritmo da pujante nação. Nas fases seguintes, em estreita relação com a política cultural instituída por Victoria Ocampo a partir de 1931 por meio da revista Sur e o seu posterior projeto editorial, a tradução foi principalmente uma prática de escrita que teve uma influência decisiva na trama da literatura nacional, favorecendo sua renovação e incentivando as versões de tradutores argentinos. Esta abertura favoreceu, durante a segunda metade do século, no contexto da recepção da literatura universal, o ingresso da literatura italiana na Argentina. O presente trabalho trata de maneira sistemática, mas provisória, o panorama da literatura italiana traduzida na Argentina. $O$ objetivo principal comporta o registro dos momentos mais fecundos e a interpretação dos resultados obtidos em relação à tradução no sistema literário nacional.

PALAVRAS-CHAVE: literatura italiana, literatura argentina, tradução, fase de desenvolvimento.

ABSTRACT: In less than two centuries of Argentinian literature, the development of translated Italian literature is intimately related to the pedagogical or aesthetic aims determined by the cultural context of the different historical periods. In general terms, it is possible to assert that literary translation in Argentina had as its aim the expansion of the cultural horizon of a reader that consolidated itself along with the new nation. Translation was a writing practice with decisive influence on national literature during the subsequent stages and it was closely related with the cultural policy carried out by Victoria Ocampo from 1931 onwards by means of the magazine Sur and its further editorial project. This was due to the fact that it favoured its renewal and incentivated numerically and quantitatively the versions from national translators. The present study proposes a systematic but provisory panorama of Italian literature translated in Argentina. The main objective is to record the most abundant moments and the interpretation of the results obtained in 
relation to translation in the national literary system.

KEYWORDS: Italian literature, Argentine literature, translation, development stages. 
Il presente lavoro tratta in maniera sistematica, ma provvisoria, il panorama della letteratura italiana tradotta in Argentina. Lo scopo principale, vale a dire quello di delimitare una cartografia che faciliti la sua organizzazione, comporta il registro dei momenti più fecondi e l'interpretazione degli esiti ottenuti nei confronti della traduzione nel sistema letterario nazionale. Visto il carattere versatile che la traduzione di opere letterarie ha in questo paese, è questo - senza dubbio - un obiettivo difficile da raggiungere, per cui si prevedono tappe di indagine successive nelle quali potranno includersi, oltre ai registri quantitativi, le valutazioni particolari delle opere tradotte, le quali sono considerevolmente aumentate nella seconda metà del Novecento, e lo studio degli specifici contesti di produzione. Ciononostante, e a prescindere dalla magnitudine dell'oggetto, è fattibile in quest'occasione definire inquadrature e segnare cronologie possibili.

Intesa come la riscrittura di un testo originale in una seconda lingua, la traduzione letteraria può essere pensata come una diaspora della scrittura che consente la disseminazione e permette a un testo originale di germinare in una nuova lingua (Romano Sued, 2014, p. 65). L'idea di un originale disseminato in molteplici versioni e quella di un traduttore che interpreta le motivazioni estetiche di questo originale costituiscono le tensioni principali di questo particolare atto di creazione. Il noto aforisma italiano traduttore, traditore avverte appunto la dicotomia che la traduzione letteraria comporta. La libertà del traduttore di trovare nella lingua meta gli effetti analoghi che l'autore ha proposto nella lingua di partenza è condizionata dall'idea di 
un originale che bisogna proteggere. La traduzione letteraria mostra, pertanto, aspetti della creazione letteraria che uniscono la conoscenza tecnica di una lingua con l'intuizione estetica di un enunciatore. Così il traduttore letterario, dotato di una particolare coscienza linguistica, elabora un proprio modo di esplorare, cercare ed esperimentare nella sua lingua; la sua è una pratica che esalta la funzione estetica e arricchisce il sistema letterario di arrivo, allargando le sue possibilità espressive e favorendo l'impulso di universalità che ogni letteratura nazionale insegue. La traduzione letteraria definisce, quindi, un nuovo atto di scrittura che mette in valore le molteplici conoscenze del traduttore. È la singolarità di questa coscienza linguistica quella che cerca di dire quasi la stessa cosa nel delicato passaggio che comporta la migrazione da una lingua all'altra.

Rilevare e interpretare la traduzione di opere letterarie nell'orizzonte del sistema letterario nazionale implica determinare un criterio di ricerca che metta in rapporto tutti i fattori che su essa influiscono. Per questo è specialmente appropriato ricorrere alla nozione di costellazione, perché consente di studiare la traduzione letteraria come un "conjunto de puntos fulgurantes en el horizonte del sistema literario nacional" (Willson, 2004, 229). Così come il corpo astronomico regola la sua luminosità con l'energia dei propri astri, questo insieme di traduttori definisce l'intensità della sua luce con il ritmo e la qualità delle loro versioni. Tuttavia, e contrariamente all'immobilità che gli astri dimostrano alla percezione umana nell'universo, le costellazioni letterarie scintillano al ritmo del tempo storico e splendono conforme alle trasformazioni contestuali che operano nel campo artistico e culturale.

Come abbiamo detto in un lavoro precedente sulle relazioni tra la letteratura argentina e quella italiana (Blanco, Cattoni e altri; 2008, p. 290), la questione delle traduzioni di opere in Argentina è abbastanza complessa, per diversi fattori. Il fattore fondamentale risiede nella varietà di spagnolo che si utilizza. Il problema più evidente è che solo una parte delle traduzioni pubblicate nel nostro paese è fatta da traduttori argentini e nella varietà rioplatense.

In due secoli di storia della letteratura argentina, lo sviluppo della letteratura italiana tradotta è in stretto rapporto con i propositi pedagogici o estetici determinati dal contesto culturale dei diversi momenti storici. In linea di massima, è possibile affermare che, dalla conformazione dello Stato nazionale e durante le prime decadi del XX secolo, la traduzione letteraria mirava ad ampliare l'orizzonte culturale di un lettore che si consolidava al ritmo della fiammante nazione. Le collane di letteratura straniera pubblicate agli inizi del novecento, nella maggior parte versioni spagnole ignare delle norme della varietà rioplatense, inseguivano un proposito, 
anziché estetico, pedagogico. Nelle fasi successive e in stretto rapporto con la politica culturale portata avanti da Victoria Ocampo a partire dal 1931 tramite la rivista Sur e il suo posteriore progetto editoriale, la traduzione è stata soprattutto una pratica di scrittura che ebbe un'influenza decisiva nell'ordito della letteratura nazionale favorendo il suo rinnovo e incentivando le versioni di traduttori argentini. L'ideale di universalismo e cosmopolitismo moderno che la direttrice di questa pubblicazione ha voluto per le lettere argentine trovò nella traduzione letteraria nuovi modi di rappresentazione e anche nuovi materiali e modelli di composizione. Non vi sono dubbi che il progetto editoriale promosso da questa prestigiosa rivista segnò un precedente nella storia delle traduzioni letterarie nel paese. Fu questa un'apertura che favorì, durante la seconda metà del secolo, nel contesto della ricezione della letteratura universale, l'ingresso della letteratura italiana in Argentina.

II. È possibile realizzare un breve percorso per questa storia delle traduzioni letterarie italiane prendendo come punto di riferimento appunto la rivista Sur. In questa particolare cartografia, la mappa del territorio della traduzione italiana in Argentina comincia a delimitarsi a partire da certi fatti nel tempo, il che ci consente di distinguere in qualche modo le diverse tappe o periodi, e riconoscerne i traduttori di rilievo.

Molto prima dell'uscita di Sur, dall'indipendenza dalla Spagna fino al consolidarsi del progetto nazionale, la traduzione di opere letterarie italiane rispose a motivazioni politiche e pedagogiche. Per questo, rivestono speciale interesse nell'ambiente letterario degli autori italiani che preconizzarono e consolidarono ideali del Risorgimento. Motivazioni patriottiche, conformi al sentimento romantico nazionale dell'epoca, portano il cordobese José Antonio Miralla a tradurre Foscolo, Esteban de Luca e Juan Cruz Varela a tradurre Alfieri e Juan María Gutiérrez a tradurre Manzoni.

Tuttavia, in quest'orizzonte, è possibile rintracciare delle iniziative - come quella di Bartolomé Mitre - che orientano la traduzione letteraria verso un indirizzo estetico. Nel 1897 , dopo una decade di lavoro, Mitre offre la prima traduzione argentina della Divina Commedia di Dante Alighieri, alla quale allega una personale Teoria della traduzione. Benché questa sia stata una versione con alcune limitazioni, il suo valore risiede non solo nell'importanza e lunghezza dell'opera, ma nel fatto che per la prima volta si presenta nel panorama letterario argentino una riflessione teorica sulla traduzione letteraria.

Questo periodo si conclude con la traduzione che Leopoldo Lugones fece del poema $L a$ 
calandria di Pascoli, e con versioni di poemi e racconti di D’Annunzio, eseguite da Leopoldo Díaz e Horacio Quiroga.

A partire dalla metà degli anni '30 fino alla fine degli anni '50, nell'ambito editoriale dell'America Latina, fu precisamente la centralità di Buenos Aires a favorire una forte politica di edizione che incentivò in maniera straordinaria la traduzione letteraria e consolidò le sue finalità estetiche. Sur fu, senza dubbio, una necessaria iniziativa culturale che incorporò la letteratura straniera al sistema letterario nazionale tramite una definita politica di traduzione che privilegiò le versioni argentine piuttosto che quelle spagnole. A partire da questo momento, l' Argentina creò le condizioni per la sua epoca d'oro della traduzione. Nonostante le differenze esistenti tra gli intellettuali di Sur, essi rinnovarono il catalogo di traduzioni e, pertanto, nuovi modi di rappresentazione e anche nuovi materiali e modelli di composizione sono entrati nel sistema letterario nazionale. La messa in valore della traduzione fu conseguenza di una nuova concezione di letteratura e, quindi, di traduzione letteraria, intesa ora come una pratica di scrittura che opera tra due lingue e consente nuovi rapporti tra la letteratura straniera e la letteratura nazionale. È per questo che, a partire da allora, la traduzione letteraria verrà sviluppata fondamentalmente da scrittori o traduttori con una solida e sofisticata formazione letteraria, e quindi, agenti riconoscibili nel campo letterario nazionale.

Benché in numero minore rispetto ad altre letterature europee, le lettere italiane trovarono nella rivista e nel suo progetto editoriale un mezzo di diffusione. Quantunque solo il volume 225, nel 1954, sia dedicato interamente alla letteratura italiana, a partire dalla seconda metà degli anni '30 è possibile rintracciare traduzioni di opere italiane. Così, nell'agosto del 1936, la rivista pubblica le traduzioni di Ernesto Palacios di due poemi di Ungaretti e, nel 1949, una traduzione anonima di Conversazioni in Sicilia di Elio Vittorini e la versione eseguita da Enrique Pezzoni di La gazzetta nera di Guido Piovene.

L'impatto nel settore editoriale del progetto Sur generò nel campo culturale il sorgere e il consolidarsi di case editrici quali Losada, Sudamericana, Emecé, Compañía Editorial Fabril, Nueva Visión, tra altre, le quali, a partire dagli anni '60, aumentano le pubblicazioni di traduzioni di opere italiane. Autori contemporanei di rilievo quali Svevo, Pirandello, Moravia, Montale, Quasimodo, Silone, Pavese, Pratolini, Vittorini, Gadda, Pasolini, Morante e Calvino, insieme all'intervento della gestione editoriale di notevoli operatori culturali (come E. Pezzoni in Sudamericana, noto traduttore tanto dall'inglese quanto dall'italiano, o come Edgar Bayley, direttore della collana di letteratura della casa editrice Nueva Visiòn), veicolarono il lavoro di 
traduttori di prestigio come Tristán Suárez (pseudonimo utilizzato da Carlos Liacho), Horacio Armani, Atilio Dabini, Rodolfo Alonso e Aurora Bernárdez in versioni canoniche. Inoltre, in quegli anni, la sinistra contribuì alla diffusione di autori emblematici, come Antonio Gramsci, tradotto da José Aricó e Héctor Agosti.

Negli anni '70, ancora in questo contesto di apogeo editoriale, spicca la collana "Biblioteca Básica Universal” del Centro Editor di America Latina. Dei 253 titoli del catalogo, 28 corrispondono a opere di letteratura italiana, molte delle quali furono pubblicate per la prima volta nel paese. Notevoli sono il Novellino, di autore anonimo, le cronache di Giovanni Villani e Dino Compagni. Tra i titoli di questa collana spiccano specialmente le antologie perché favorirono la divulgazione di classici rinascimentali e moderni di scarsa diffusione nel paese. È specialmente significativo in questo progetto il lavoro di Leopoldo di Leo, il quale selezionò, tradusse e prologò molti dei volumi di questa collana, come i Cantos órficos y otros cantos [I canti orfici e altri canti] (che include testi di Campana, Saba, Ungaretti, Montale, Quasimodo), Los mares del sud y otros poemas [I mari del sud e altri poemi] (con poesie di Pavese, Pasolini e altri), Pelirrojo alimaña y otros cuentos [Rosso malpelo e altri racconti] (con racconti di Verga, Pirandello, Moravia e altri), Poesía Medieval italiana [Poesia medievale italiana] e Prosistas medievales italianos [Romanzieri medievali italiani] (che include scrittori come Franco Sacchetti, Sercombi e altri).

In questa prospettiva cronologica, merita una speciale considerazione la traduzione di poesie. Una letteratura come quella italiana, che fece della poesia la sua manifestazione più genuina, suscitò in poeti e traduttori argentini, da sempre, un costante fascino. Quella memorabile iniziativa di Mitre trova, a partire da questo momento, una risonanza degna di nota. Così, nel 1979, la casa editrice Lohle pubblica la versione bilingue della Divina Commedia, tradotta dal noto filologo Ángel Batistessa e, recentemente, nel 2015, la casa editrice Edhasa ha fatto altrettanto con la versione del poeta Jorge Aulicino.

Per i motivi summenzionati, la seconda metà del secolo scorso fu prodiga di versioni di poesia. Traduttori autorevoli organizzarono antologie di poeti contemporanei che meritano attenzione. Il volume Poesía Italiana Contemporánea inaugura questa serie e mette in risalto il valore della traduzione di Alberto Girri e Viola Soto, i quali hanno saputo scoprire la rilevanza di poeti quali Saba, Cardarelli, Ungaretti, Montale, Quasimodo, Penna, Sinisgalli, Pavese, Gatto e Sereni. Horacio Armani ha pubblicato tre antologie fondamentali: nel 1971 Antología de Eugenio Montale [Antologia di Eugenio Montale], per la prima volta pubblicato in spagnolo; 
nel 1973 Poetas Italianos del Siglo XX [Poeti italiani del XX secolo] edito nel 1974 da Lautaro e poi riedito da Ediciones Unesco con il titolo Poesía Italiana Contemporánea [Poesia italiana contemporanea], e El vacío que nos invade [Il vuoto che ci invade], una selezione di poemi di Montale pubblicata nel 1990. Per la prima volta, un insieme di 29 poeti, un totale di 124 poemi tradotti e un significativo apparato critico furono a disposizione del lettore ispano-americano. Sono anche da nominare le due antologie e traduzioni di Antonio Aliberti: Un siglo de poesía italiana [Un secolo di poesia italiana] (1891-1997), (Buenos Aires, Ocruxaves, 1977) e La poesía italiana en el tiempo (del Medioevo hasta nuestros días) [La poesia italiana nel tempo. Dal Medioevo fino ai giorni nostri], (Buenos Aires, Autel, 1999). Alle succitate collane, si aggiunge Poesía italiana Contemporánea [Poesia italiana contemporanea] di Rodolfo Alonso, edita dall'Università di Carabobo nel 1971. Negli ultimi anni, spiccano da una parte il lavoro di Adriana Crolla La piel desnuda. Poetas italianas entre milenios [La pelle nuda. Poetesse italiane tra i millenni] (Rosario, Casa Editrice Laborde, 2001) e il lavoro di Diego Bentivegna, Viaggio in Italia. 8 poetas italianos contemporáneos (ensayo-traducción) [Viaggio in Italia. Otto poeti italiani contemporanei] (Casa Editrice Sigamos enamoradas, Buenos Aires, 2009).

Alle antologie si aggiunge inoltre la traduzione di opere individuali di poesia italiana, la cui enumerazione eccede la portata di questo lavoro. Tuttavia, è bene anche menzionare le versioni di Montale e Pavese che offrirono Armani e Alonso, quelle che Oreste Fratoni fece dell'opera di Giuseppe Ungaretti, la traduzione di Leopardi e dell'ultimo Ungaretti eseguita da Ricardo Herrera, le versioni di Mario Luzi a cura di María Julia De Ruschi Crespo, quelle di Ungaretti y Montale di Elena Tardonato Faliere, quelle di Alda Merini, a cura di Delfina Muschietti e la recente versione di Vita Nuova - Vida Nueva di Dante eseguita da R. Raschella e pubblicata dalla casa editrice Santiago Arcos.

Per quanto riguarda altri generi, negli ultimi anni, spiccano in narrativa: Roberto Raschella, traduttore di N. Machiavelli, G.Verga, L. Pirandello, I. Svevo, G.Della Volpe, C. Pavese,G. Rodari; Guillermo Piro e Eugenio Montequin, di J.R.Wilcock; e Alejandro Patat, il quale ha recentemente tradotto le Operette morali di G. Leopardi. Una menzione speciale meritano le notevoli versioni di classici rinascimentali tradotte in questi ultimi anni da Nora Sforza. $L a$ Cassaria di L. Ariosto, Il Candelaio di G. Bruno pubblicate dalla casa editrice Libros Raros, la prosa di N. Machiavelli riunita in Ficción completa [Narrativa completa] pubblicata da Colihe e anche Seis personajes en busca de autor [Sei personaggi in cerca di autore] di L. Pirandello.

La saggistica è diffusa da note case editrici come Cuenco de Plata, la quale, grazie all'accurato 
impegno di Edgardo Ruso, incluse nel catalogo testi di classici, quali Tommaso Campanella e Torquato Accetto, e anche di contemporanei, come Remo Bodei. Va sottolineata la recente traduzione che questa casa editrice ha fatto di La Divina Mimesis di Pasolini, tradotta da Diego Bentivegna, il quale si è anche occupato di Antonio Gramsci. Una delle tante piccole case editrici che sono sorte negli ultimi dieci anni di questo secolo, la casa editrice Winograd, è responsabile della traduzione di opere di Pico della Mirandola e la traduzione del Convivio di Dante eseguita da Mariano Pérez Carrasco ed edita da Colihue. Questo elenco che sicuramente non è completo tenta soltanto, come detto in precedenza, di offrire un panorama generale, quale mappa orientativa che non riesce mai a descrivere tutti i dettagli del rilievo.

III. Nell'attualità, la ristrutturazione del mercato editoriale nel contesto della globalizzazione economica determina che l'affluenza di versioni dipenda ancora una volta dalla Spagna. Come avverte J. Aulicino (2013) i grandi traduttori argentini oggi non traducono per le grandi case editrici. Il mercato dell'editoria nazionale è dominato da tre grandi case editrici non argentine: Planeta, Santillana e Penguin Random House Grupo Editorial, le quali hanno annesso Sudamericana ed Emecé. I traduttori argentini più riconosciuti e esperti non lavorano oggi, in generale, per i grandi editori che dominano il mercato ispano. Inoltre, devono affrontare il problema dei diritti d'autore che, nella maggior parte, corrispondono alle case editrici spagnole. Come si evince dalla precedente descrizione, "la Argentina tuvo una edad de oro de la industria editorial y, con ella, de la traducción. No es esta" (Aulicino; 2013). La sorte che corrono gli autori argentini nel paese è in stretto rapporto con le politiche di diffusione nel mercato dell'editoria, guidato - naturalmente - dalla Spagna e gli autori che oggi hanno successo nella penisola come Andrea Camilleri, pubblicato dalla casa editrice Salamandra e nella varietà dello spagnolo di Madrid. Dentro un cerchio più stretto di lettori, il caso di A. Tabucchi e A. Baricco, entrambi pubblicati da Anagrama, vengono anch'essi letti in una varietà di lingua estranea al lettore argentino. Si diffondono anche nomi di saggisti e filosofi, come Umberto Eco, pubblicato da Lumen, Gianni Vattimo, Toni Negri e Giorgio Agamben. In Argentina non esistono case editrici specializzate in letteratura italiana e neppure un lavoro di esplorazione che identifichi autori d'interesse e li traduca. In questo nuovo disegno globale, è la Spagna quella che possiede i diritti di gran parte degli scrittori italiani.

Il fatto che in Argentina non vi sia un numero significativo di traduzioni locali attira l'attenzione, soprattutto se prendiamo in considerazione che è un paese che ha costruito gran 
parte della sua identità culturale nella traduzione e più del $50 \%$ della popolazione è di origine italiana. Questi due aspetti non hanno garantito la conservazione dell'italiano come lingua di cultura e quindi un ritmo costante di traduzioni letterarie che consentisse tra entrambi i paesi un vincolo culturale. Forse è conveniente segnalare al riguardo che la maggior parte degli immigrati che sono arrivati nel paese, contadini poveri in cerca di migliori condizioni di vita, ebbero con l'italiano un rapporto di estraneità. La tardiva unificazione politica italiana determinò anche una tardiva unificazione linguistica. Soltanto le classi più avvantaggiate, che non immigrarono, si vantavano del dominio dell'italiano. Per la maggior parte degli immigrati italiani, l'italiano non era la loro lingua di cultura. I contadini, quelli che in maggior misura sono immigrati, utilizzavano le diverse varietà dialettali e, una volta nel paese di arrivo, restrinsero quella varietà all'uso privato dell' ambito familiare. Il noto scrittore Manuel Puig, riguardo alle tradizioni degli immigrati italiani, afferma: "La masa de la población argentina fue formada por la inmigración de principios de siglo, sobre todo los italianos, y esos campesinos que llegaron para cambiar de status eran gente que venía a olvidar sus tradiciones, no a continuarlas" (D. Torres Fierro, 2014). Come impatto linguistico e visto l'elevato indice di italianismi che si osservano nello spagnolo del Río de la Plata, quanto verificatosi nella società argentina rispetto all'italiano fu un fenomeno di interferenza piuttosto che di conservazione.

Insomma, nell'attualità il mercato editoriale che regola la distribuzione di libri in Argentina dipende praticamente dalla Spagna e questo determina la varietà linguistica adoperata nelle versioni, che non è certamente la varietà del Rio de la Plata. Inoltre, le grandi case editrici non assumono i noti traduttori e, infine, la mancanza di buone politiche di diffusione fanno sì che il pubblico argentino non s'interessi di letteratura italiana. Il panorama italiano contemporaneo è scarsamente diffuso dai grandi giornali. Non esistono neanche giornalisti critici con un'accurata conoscenza del panorama culturale italiano. Tutti questi fattori spiegano perché la letteratura italiana non abbia una presenza di risalto nelle librerie argentine. Benché, in genere, in Argentina gli scrittori italiani abbiano avuto una buona ricezione, questo non significa che la letteratura italiana sia quella più diffusa. Pirandello, Ungaretti, Calvino, scrittori canonici che sono venuti in Argentina, sono stati letti ma a stento hanno portato alla lettura di altri scrittori. La letteratura italiana attuale si muove in circuiti ridotti o iniziative accademiche che non incidono sul grande pubblico.

IV. Fin qui questa specie di cartografia storica ha tentato di riassumere i diversi momenti 
attraversati dalla letteratura italiana tradotta nel panorama generale argentino fino allo stato attuale. Tuttavia, non si tratta di un territorio uniforme e regolare: vi è una zona, Córdoba, che, almeno dagli ultimi anni del XX secolo fino all'attualità, spicca nella mappa per le sue particolari caratteristiche. Nel contesto nazionale, Córdoba possiede una considerevole attività di traduzione di letteratura italiana, stimolata fondamentalmente dall'ambito universitario.

In ordine cronologico, risalta la collana Il nuovo e vechio stil, a cura della professoressa Trinidad Blanco de García, la quale a partire dal 1984 ha offerto una serie di traduzioni di poeti italiani e alcune versioni all'italiano di poeti argentini. La collana è stata conclusa con tre volumi dedicati ai poeti Dino Campana, Umberto Saba e Giuseppe Ungaretti, pubblicati nel 1985, 1987 e 1991, ai quali, oltre alla professoressa Blanco de García, hanno collaborato i traduttori Horacio Armani, Ricardo Herrera e Pablo e Esteban Anadón. Nel 1997, la rivista "Azzurra" dell'Istituto Italiano di Cultura a Córdoba, ha pubblicato, nel suo anno IV, i numeri 10/11/12 in un volume solo con il titolo Recorridos de la poesía italiana del siglo XX [Percorsi della poesia italiana del XX secolo], che costitù̀ una vera antologia di 25 poeti italiani contemporanei in versione bilingue. Per quanto riguarda la poesia, va menzionata l'antologia di Pablo Anadón El astro disperso. Últimas transformaciones de la poesía en Italia [L'astro disperso. Ultime trasformazioni della poesia in Italia] (1971-2000), pubblicata dalla casa editrice Del Copista, nel 2001. Trinidad Blanco de García ha anche tradotto Obritas Morales [Operette morali] di Leopardi nel 2000; Silvio Mattoni ha tradotto una versione di La luna y las fogatas [La luna e i falò] di Cesare Pavese nel 2003; e infine, Esteban Nicotra ha tradotto Empirismo Herético, di Pier Paolo Pasolini nel 2005. Di recente, la casa editrice La Sofía Cartonera, dalla sua collana Traiciones Cartoneras, ha offerto una traduzione di alcuni racconti di Adrián Bravi con il titolo La línea del Ecuador [La linea dell'equatore] e sta preparando il volume Rimas [Rime] de Michelangelo Buonarroti in collaborazione con la università di Bahia Blanca e il volume Pensamientos [Pensieri] di Leopardi. In quest'ultimo caso, parliamo di traduzione collaborativa, in quanto i traduttori sono molti: un gruppo di laureati della Facoltà di Lingue ed altri appartenenti alla cattedra di Letteratura Italiana della Facoltà di Filosofia ed Umanistica.

Lo studio fin qui proposto ha avuto come obiettivo facilitare l'organizzazione dell'enorme quantità di materiale corrispondente alla traduzione della letteratura italiana nel sistema letterario argentino. Come qualsiasi cartografia agli inizi, ha un carattere provvisorio, inconcluso... Semplici segni su una mappa, alcuni fatti, alcune referenze indispensabili, altre orientative, alcuni punti folgoranti in una costellazione e, senza dubbio, molte omissioni e zone ancora 
da delimitare. Il lavoro dei cartografi è sempre incompleto e in ciò risiede, appunto, il suo appassionante interesse: l'invito costante a nuove esplorazioni.

\section{Riferimenti bibliografici}

AULLICINO, J.“¡Qué viva la traducción!- La letteratura italiana in Argentina” http://www. nazioneindiana.com/2013/06/01/que-viva-la-traduccion-la-letteratura-italiana-in-argentina/ Aceso14/02/2016.

BLANCO T, CATTONI, S. y otros. Repertorio Bibliográfico de las relaciones entre las Literaturas Argentina e Italiana. Córdoba: del Copista, 2008.

BLANCO, T. Italia en el imaginario de los escritores argentinos, (Premio Fondo Nacional de las Artes). Córdoba: Garden Press, 1995.

BRUSCHI, R. Le edizioni argentine di Fontamara negli anni '30 In ARTUCIO G. (org.) Escrituras e Imágenes. Paraná: editorial Uader, 2014, p.394.

GIANCIOTTI, V. (a cura di) La bibliografía della letteratura italiana in America Latina. Torino: Edizioni della Fondazione Giovanni Agnelli, 1992.

TORRES FIERRO, D. “Conversación con Manuel Puig: La redención de la cursilería. Entrevista a Manuel Puig', en http://www.revistacontratiempo.com.ar/puig.htm. Aceso 9/11/2014.

ROMANO SUED, S. "El dilema de la traducción. El viaje y la diáspora de la escritura”. http://www. raco.cat/index.php/Transfer/article/viewFile/287005/375218. Aceso 20/9/2016

WILLSON, P. La constelación del sur: traductores y traducciones en la literatura argentina del siglo XX. Buenos Aires: Siglo Veintiuno Editores, 2004.

Recebido em 12/12/2016

Aprovado em 15/04/2017 